\title{
Real-World Field Performance of the Los Angeles Motor Scale as a Large Vessel Occlusion Screen: A Prospective Muticentre Study
}

\author{
Tej G. Stead ${ }^{a}$ Paul Banerjee ${ }^{b, c, d}$ Latha Ganti ${ }^{b, c, d}$

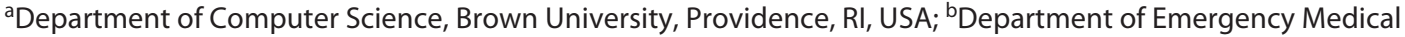 \\ Services, Polk County Fire Rescue, Bartow, FL, USA; ' Department of Emergency Medicine, Envision Physician \\ Services, Plantation, FL, USA; ${ }^{\mathrm{d} D e p a r t m e n t}$ of Clinical Sciences, University of Central Florida, Orlando, FL, USA
}

\section{Keywords}

Los Angeles motor scale · Acute stroke · Emergency medical services - Prehospital

\begin{abstract}
Background: The Los Angeles Motor Scale (LAMS) is a 3-item, 0-to-5-point motor stroke-deficit scale derived from the Los Angeles Prehospital Stroke Screen. We assessed the predictive validity (for interventions performed and discharge disposition) of the LAMS performed in the field by paramedics in a geographic region of over $5,200 \mathrm{~km}^{2}$, covering both rural and urban areas. Methods: We analyzed data gathered from Phase I of the LIT-PASS study (Large Vessel Occlusion Identification Through Prehospital Administration of Stroke Scales) which included all patients with suspected acute cerebrovascular disease, as assessed by the Balance, Eyes, Face, Arm, Speech, Terrible Headache/Time to Call 911 (BE-FAST) test. Results: Among 1,906 patients with median age 72 years (interquartile range [IQR] 60-81), 53\% were female with a median on-scene time of $15 \mathrm{~min}$ (IQR 12-19). C statistics for the interventions of mechanical thrombectomy, alteplase administration, computed tomography angiography, and perfusion imaging were $0.681,0.643$, and 0.680 , respectively. The cut point for predicting these 3 interventions was confirmed to be LAMS $\geq 4$. LAMS $\geq 4$ had sensitivity 0.730 ( $0.661-$ $0.790)$ and specificity $0.570(0.539-0.601)$ for mechanical in-
\end{abstract}

tervention (endovascular thrombectomy, coiling, or clipping) and relative risk of 2.98 (2.19-4.07) for in-hospital death. Conclusions: This real-world field study validates the LAMS as an effective tool for prehospital assessment of suspected strokes in determining transport decisions, with predictive validity for interventions performed.

(c) 2021 The Author(s).

Published by S. Karger AG, Basel

\section{Introduction}

Acute ischemic stroke is the fifth leading cause of death and the number one cause of disability [1]. The only acute guideline-recommended interventions to date are thrombolytic therapy with intravenous alteplase $[2,3]$, and endovascular thrombectomy [3]. Alteplase can be administered up to $4.5 \mathrm{~h} \mathrm{[4]} \mathrm{after} \mathrm{symptom} \mathrm{onset,} \mathrm{and} \mathrm{endovas-}$ cular thrombectomy can be performed up to $24 \mathrm{~h}$ of stroke symptoms onset [5]. However, both treatments have been shown to be most effective the sooner after stroke onset they are administered [6,7]. Because of the time-sensitive nature of these treatments, the role of emergency medical services personnel is a critical link in the stroke chain of survival [8].

With the advent of specialized stroke center designations such as Comprehensive Stroke Centers [9, 10], Thrombectomy-capable Stroke Centers, and Primary karger@karger.com www.karger.com/ced

Karger $\stackrel{\text { ' }}{5}$

BOPEN ACCESS
(C) 2021 The Author(s)

Published by S. Karger AG, Basel

This is an Open Access article licensed under the Creative Commons Attribution-NonCommercial-4.0 International License (CC BY-NC) (http://www.karger.com/Services/OpenAccessLicense), applicable to the online version of the article only. Usage and distribution for commercial purposes requires written permission.
Correspondence to:

Latha Ganti, lathagantimd@gmail.com 
Stroke Centers the prehospital triage process became more complex. Furthermore, 10\% of strokes are hemorrhagic [1], a subset of which are amenable to interventions such as aneurysmal coiling, or clipping. A study of over 36,000 hemorrhagic stroke patients found that they were more likely to receive neurosurgical and endovascular treatments and be alive at 90 days when admitted to comprehensive stroke centers than patients admitted to other hospitals [11]. This is yet another layer of complexity. Defining mechanical intervention as endovascular thrombectomy, coiling, or clipping, the ability to perform mechanical intervention is what distinguishes a comprehensive stroke center from a thrombectomy-capable stroke center. By contrast, a primary stroke center can only provide alteplase for ischemic strokes, and supportive treatment for intracranial hemorrhage. The triaging decision of where to transport is complex, affecting patients, emergency medical services systems, and hospitals. The bypassing of a primary stroke center for a comprehensive stroke center means that the primary stroke centers' volume might decrease to a level where it may no longer be able to maintain its expertise. For the patient, it could mean a delay in receiving alteplase, which is currently still indicated for both large vessel occlusion and nonlarge vessel occlusion strokes if within $4.5 \mathrm{~h}$ of onset. Bypassing a primary stroke center to travel to a comprehensive stroke center would take an ambulance out of commission for a longer period of time, which could be detrimental to small communities that may have a limited ambulance fleet and personnel. It could mean that the next stroke patient would have to wait longer to get treatment, and potentially miss treatment time windows. On the other hand, the comprehensive stroke center that receives all patients due to indiscriminate primary stroke center bypass could become overwhelmed, with not enough neuro intensive care unit beds to accommodate all the transports. It is well known that emergency department boarding is bad for patient outcomes [12-15] and this problem would be exacerbated in busy hospitals that are also comprehensive stroke centers.

Given the above, it is imperative that emergency medical services be able to identify large vessel occlusions in the field. In order to do so, several scales have been proposed and studied. Four systematic reviews [16-19] reported on the accuracy of prehospital scales for field detection of large vessel occlusion and concluded that no one scale was especially superior but that more studies were necessary as the included studies were limited to retrospective derivations of studies done on cohorts of known strokes. Furthermore, the included studies were

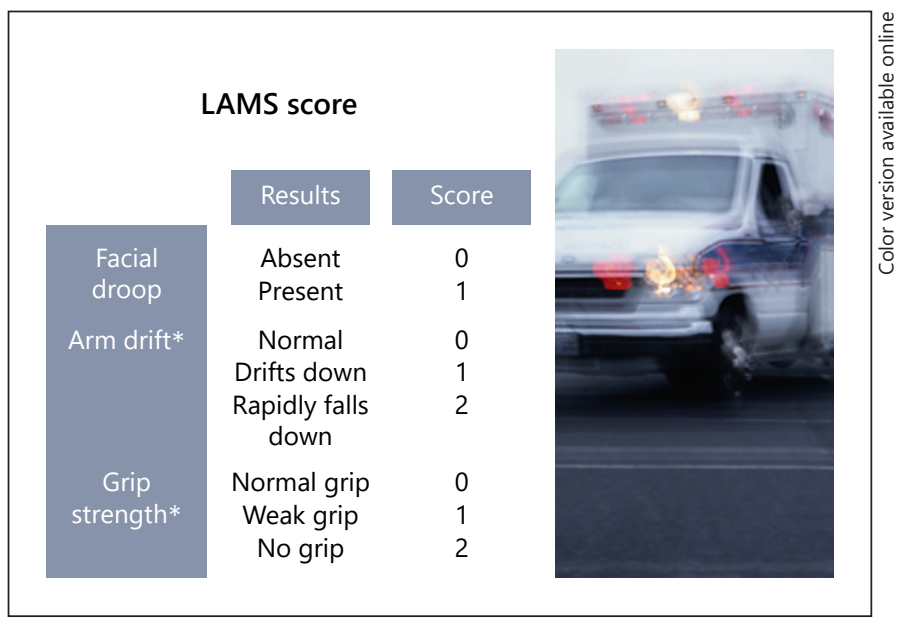

Fig. 1. LAMS score. LAMS, Los Angeles Motor Scale.

small, with the large vessel occlusion screen done mostly by nurses, emergency department physicians, or neurologists, and usually upon emergency department arrival. This is not representative of the actual field experience, where the population consists of suspected (rather than confirmed) strokes, and the scales are performed by paramedics rather than nurses or physicians.

Polk County Fire Rescue is one of the largest emergency medical services systems in our state, responding to more than 115,000 calls per year, and covering a geographic footprint of over 2,010 square miles. In this paper, we describe our real-world experience using the Los Angeles Motor Scale (LAMS) in our emergency medical services system. The LAMS is a 3-item, 0 - to 5 -point motor deficit scale, derived from the Los Angeles Prehospital Stroke Screen. The scale assesses facial droop, arm drift, and grip strength [20] and is described in Figure 1. The primary aims of this study were (1) to report the sensitivity and specificity of the LAMS in predicting which patients needed a comprehensive stroke center, and (2) to calculate which LAMS score affected bypass decisions.

\section{Methods}

Protocol

The Polk County Fire Rescue Phase I stroke protocol begins with the BE-FAST. If it is $>0$, then time of onset is obtained. If it is $<24 \mathrm{~h}$, then a modified Rankin score (mRS) is obtained. If the mRS is $<3$, then anticoagulant screening is done. The anticoagulant screening is to ensure that patients who have contraindications to alteplase do not potentially waste time by going to a Primary stroke center first. Finally, a LAMS is performed, and if $\geq 4$, then the patient is transported to a comprehensive stroke center. In other 
Fig. 2. Polk County Fire Rescue stroke protocol for LIT PASS (LVO Identification through Prehospital Administration of Stroke Scales) Phase 1.

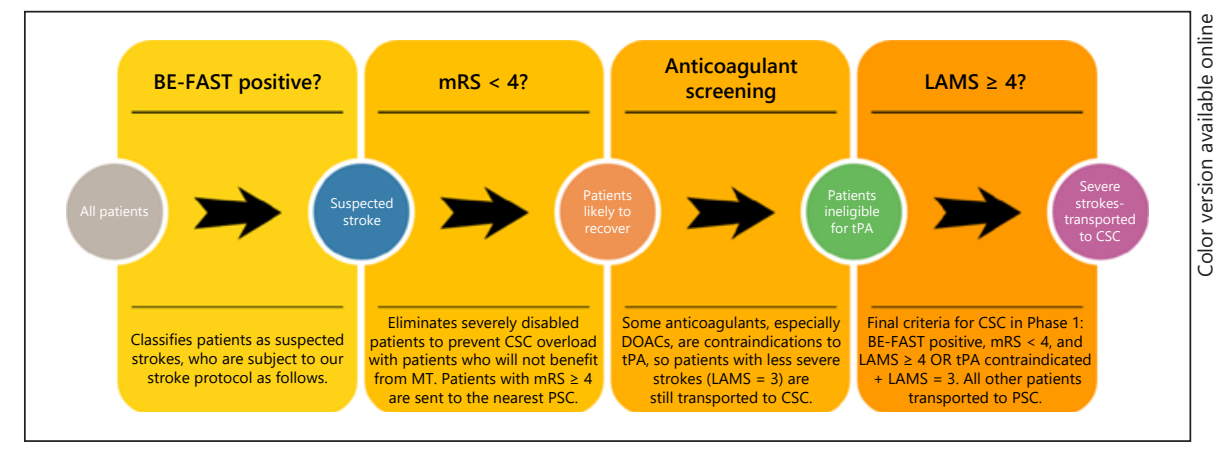

words, patients went to a comprehensive stroke center if they either had LAMS 4 and $\mathrm{mRS}<3$, or they had a LAMS $=3$ and $\mathrm{mRS}$ $<3$ but had a contraindication to alteplase. Figure 2 illustrates our Phase 1 stroke protocol [21]. To reduce the risk of selection bias, every patient transported for suspected stroke (defined as BEFAST $>0$ ) was included in the study. This better reflects the prehospital setting, where transport decisions must be made for every call.

\section{Data Collection}

Our Institutional Review Board approved prospective study included all patients transported for suspected stroke by our county emergency medical services system from January 2016 to May 2019. The primary "outcome" was any mechanical intervention, whether endovascular thrombectomy for large vessel occlusion or aneurysmal intracranial hemorrhage coiling or clipping. This was a prospective multicenter study. Our system included 6 comprehensive stroke centers, 1 thrombectomy-capable stroke center, and 4 primary stroke centers. Paramedics administered the LAMS prior to hospital arrival. Our emergency medical services system has agreements with our receiving hospitals to provide hospital discharge outcomes on all patients transported to a given facility for stroke, including the National Institutes of Health Stroke Scale at hospital arrival, hospital length of stay, and ultimate disposition of the patient.

\section{Statistical Analysis}

For all analyses, a significance level of $\alpha=0.05$ was used and all confidence intervals were $95 \%$. All correlations and hypothesis testing used nonparametric methods because the stroke scale scores are ordinal data rather than continuous. Fisher's exact test was used in place of a Pearson $\chi^{2}$ test - with small samples it may be overly conservative due to the distribution of possible $p$ values being discrete, and with large samples or numbers of categories it may require too much computational power, but neither of these were issues with our data.

In order to determine the appropriate cutoff LAMS score, we looked for its utility in predicting mechanical intervention (endovascular thrombectomy, coiling, or clipping) as these interventions can only be performed at comprehensive stroke centers, and thus influences transport decisions by our emergency medical services agency. We created a receiver operating characteristic curve to determine the cutoff point that maximizes the sum of sensitivity and specificity, and this cut-off was used for further analysis. However, we report sensitivity and specificity for all cut-off values,

Field Performance of the Los Angeles Motor Scale since certain emergency medical services systems may value sensitivity over specificity or vice versa. All statistical analyses were performed using JMP Pro 14.1 [22] for Windows.

To provide results comparable to other studies in the literature, some analyses were also performed on the subset of ischemic stroke patients, or on the subset of all stroke patients (defined as ischemic stroke, intracerebral hemorrhage, or transient ischemic attack, excluding stroke mimics, and patients with unknown diagnoses), where appropriate.

\section{Results}

There were 1,906 patients in the cohort. The median age was 72 , with an interquartile range (IQR) of $60-81$ and a range of 21-101 years. Our cohort was 53\% female. The median scene time was $15 \mathrm{~min}$, with an IQR of 12-19 min and a range of 1-74 min. Eighteen percent of the cohort had LAMS 0, 14\% had LAMS 1, 12\% had LAMS 2, $17 \%$ had LAMS 3, 16\% had LAMS 4, and $22 \%$ had LAMS 5. Forty-four percent received computed tomography angiography and computed tomography perfusion imaging, $12 \%$ received alteplase, and $13 \%$ received mechanical intervention, defined as endovascular thrombectomy, coiling, or clipping. Ten percent of strokes were hemorrhagic, $42 \%$ were ischemic, $10 \%$ were transient ischemic attacks, and $31 \%$ were stroke mimics, including seizures, migraine, sepsis, brain tumor, conversion disorder, and altered mental status. The median National Institutes of Health Stroke Scale score at hospital arrival was 7, with an IQR of 2-14 and a range of 0-40. Forty-eight percent of patients were discharged home, $19 \%$ were sent to a skilled nursing facility, $13 \%$ were discharged to rehab, and $8 \%$ expired. Further general patient characteristics, stratified by stroke type, can be found in Table 1 .

We generated 3 receiver operating characteristic curves for mechanical intervention, alteplase, and advanced imaging (computed tomography angiography and computed tomography perfusion), with area under the curve sta- 
Table 1. General characteristics of subjects, by stroke type

\begin{tabular}{|c|c|c|c|c|}
\hline Statistic & $\begin{array}{l}\text { All patients } \\
\text { (including stroke } \\
\text { mimics and unknown) }\end{array}$ & $\begin{array}{l}\text { Ischemic } \\
\text { stroke }\end{array}$ & $\begin{array}{l}\text { Intracerebral } \\
\text { hemorrhage }\end{array}$ & $\begin{array}{l}\text { Stroke } \\
\text { mimics }\end{array}$ \\
\hline$N(\%)$ & $1,906(100.0)$ & $795(41.7)$ & $187(9.8)$ & $585(30.7)$ \\
\hline Age, years, median (IQR) & $72(60-81)$ & $75(63-82)$ & $72(60-80)$ & $67(56-77)$ \\
\hline On-scene time, $\min$ & $15(12-19)$ & $14(11-18)$ & $15(12-20)$ & $16(12-19)$ \\
\hline Sex, male, $N(\%)^{*}$ & $650(47.3)$ & $283(49.6)$ & $61(48.0)$ & $178(44.6)$ \\
\hline \multicolumn{5}{|l|}{ Severity scale scores, median (IQR) } \\
\hline Prehospital LAMS & $3(1-4)$ & $3(2-5)$ & $4(2-5)$ & $2(0-4)$ \\
\hline Prehospital mRS & $1(0-2)$ & $0(0-1)$ & $0(0-1)$ & $1(0-2)$ \\
\hline NIHSS at hospital arrival & $7(2-14)$ & $9(4-16)$ & $15(8-23)$ & $4(1-10)$ \\
\hline \multicolumn{5}{|l|}{ Interventions performed, $N(\%)^{*}$} \\
\hline CTA perfusion imaging & $494(44.4)$ & $258(52.5)$ & $68(47.9)$ & $86(27.2)$ \\
\hline Alteplase administration & $206(11.6)$ & $196(25.4)$ & $0(0.0)$ & $6(1.1)$ \\
\hline Mechanical intervention & $178(15.3)$ & $156(30.2)$ & $15(10.4)$ & $6(1.8)$ \\
\hline \multicolumn{5}{|l|}{ Receiving facility type, $N(\%)^{*}$} \\
\hline Primary stroke center & $925(48.5)$ & $386(48.6)$ & $38(36.4)$ & $342(58.5)$ \\
\hline Thrombectomy-capable stroke center & $89(4.7)$ & $39(4.9)$ & $5(2.7)$ & $21(3.6)$ \\
\hline Comprehensive stroke center & $892(46.8)$ & $370(46.5)$ & $114(61.0)$ & $222(37.9)$ \\
\hline
\end{tabular}

LAMS, Los Angeles Motor Scale; mRS, modified Rankin score; IQR, interquartile range. * Percentages are reported as a proportion of the number of patients in that category with data available, which is not always equal to the total number of patients.

Table 2. Performance characteristics for all possible LAMS score cutoffs among all patients, in predicting MI

\begin{tabular}{llllllll}
\hline $\begin{array}{l}\text { LAMS score } \\
\text { cutoff }\end{array}$ & Sensitivity & Specificity & PPV & NPV & Odds ratio & Relative risk & $\begin{array}{l}p \text { value } \\
\text { (Fisher) }\end{array}$ \\
\hline$\geq 1$ & 0.944 & 0.186 & 0.174 & 0.948 & $3.85(2.00-7.43)$ & $3.35(1.81-6.23)$ & $<0.0001$ \\
$\geq 2$ & 0.893 & 0.310 & 0.190 & 0.941 & $3.75(2.29-6.15)$ & $3.23(2.04-5.11)$ & $<0.0001$ \\
$\geq 3$ & 0.820 & 0.422 & 0.204 & 0.928 & $3.33(2.22-4.98)$ & $2.85(1.98-4.10)$ & $<0.0001$ \\
$\geq 4$ & 0.730 & 0.570 & 0.236 & 0.921 & $3.59(2.52-5.12)$ & $2.98(2.19-4.07)$ & $<0.0001$ \\
$=5$ & 0.517 & 0.747 & 0.271 & 0.895 & $3.17(2.28-4.39)$ & $2.58(1.98-3.36)$ & $<0.0001$ \\
\hline
\end{tabular}

LAMS, Los Angeles Motor Scale; MI, mechanical intervention.

tistics (C statistics) of $0.681,0.643$, and 0.680 , respectively. All 3 models were significant using the likelihood ratio test, with $p<0.0001$. For predicting mechanical intervention, a cut-off of 4 had a sensitivity of 0.730 and a specificity of 0.570 , which resulted in the highest sum of sensitivity plus specificity. Accordingly, a "high LAMS" was considered to be 4 or 5 , whereas a "low LAMS" was considered to be a score of $0-3$. The scores were separated into 2 groups because the purpose of this study is to use LAMS score in order to determine transport decisions by emergency medical services personnel. However, some emergency medical services systems may require higher sensi- tivity at the cost of specificity or vice versa. To this end, we have computed the relevant statistics for all LAMS score cut-offs (Table 2). Further descriptions of patient characteristics, stratified by high/low LAMS, can be found in Table 3. To assess predictive validity of the prehospital LAMS, we calculated sensitivity, specificity, positive predictive value, negative predictive value, odds ratio, and relative risk associated with a LAMS of 4 or 5 (Table 4 ).

To assess the extent to which extent our protocol was effective and to which extent LAMS score effected bypass decisions, we examined the transport destination of all patients. We found 177 patients with a mRS $\leq 3$ and LAMS 
Table 3. General characteristics of subjects, by LAMS score category

\begin{tabular}{lcc}
\hline Statistic & LAMS 0-3 & LAMS 4-5 \\
\hline$N$ & 1,175 & 731 \\
Age, years, median (IQR) & $71(60-80)$ & $73(60-81)$ \\
Sex, male, $N(\%)^{*}$ & $401(47.0)$ & $249(47.7)$ \\
\hline Severity scale scores, median (IQR) & & $1(0-2)$ \\
Prehospital mRS & $1(0-2)$ & $14(7-21)$ \\
NIHSS at hospital arrival & $3(1-8)$ & $327(60.6)$ \\
\hline Interventions performed, $N(\%)^{*}$ & & $121(17.3)$ \\
CTA perfusion imaging & $167(29.1)$ & $130(23.6)$ \\
Alteplase administration & $85(7.9)$ & $48(7.9)$ \\
Mechanical intervention & & $223(30.5)$ \\
\hline Receiving facility type, $N(\%)^{*}$ & $702(59.7)$ & $26(3.6)$ \\
Primary stroke center & $63(5.4)$ & $482(65.9)$ \\
Thrombectomy-capable stroke center & $410(34.9)$ & \\
Comprehensive stroke center & & $377(55.5)$ \\
\hline Stroke diagnosis type, $N(\%)$ & $418(39.1)$ & $113(16.6)$ \\
Ischemic stroke & $74(6.9)$ & $37(5.4)$ \\
Intracerebral hemorrhage & $143(13.4)$ & $152(22.4)$ \\
Transient ischemic attack & $433(40.5)$ & \\
Other (stroke mimic) & & \\
\hline
\end{tabular}

LAMS, Los Angeles Motor Scale; mRS, modified Rankin score; IQR, interquartile range. * Percentages are reported as a proportion of the number of patients in that category with data available, which is not always equal to the total number of patients.

Table 4. Performance characteristics of the LAMS score in all patients, all stroke patients, and ischemic stroke patients

\begin{tabular}{|c|c|c|c|c|c|c|c|}
\hline \multicolumn{8}{|c|}{ All patients (including stroke mimics and unknown) } \\
\hline Mechanical intervention & 0.730 & 0.570 & 0.236 & 0.921 & $3.59(2.52-5.12)$ & $2.98(2.19-4.07)$ & $<0.0001$ \\
\hline CTA perfusion imaging & 0.662 & 0.655 & 0.606 & 0.708 & $3.72(2.90-4.78)$ & $2.07(1.79-2.40)$ & $<0.0001$ \\
\hline Alteplase administration & 0.587 & 0.632 & 0.173 & 0.921 & $2.44(1.82-3.28)$ & $2.19(1.69-2.85)$ & $<0.0001$ \\
\hline CTA perfusion imaging & 0.728 & 0.585 & 0.633 & 0.687 & $3.78(2.77-5.17)$ & $2.02(1.69-2.42)$ & $<0.0001$ \\
\hline Alteplase administration & 0.598 & 0.572 & 0.230 & 0.869 & $1.99(1.46-2.72)$ & $1.76(1.36-2.28)$ & $<0.0001$ \\
\hline \multicolumn{8}{|l|}{ Ischemic stroke patients } \\
\hline Mechanical intervention & 0.731 & 0.506 & 0.390 & 0.813 & $2.78(1.84-4.18)$ & $2.08(1.53-2.83)$ & $<0.0001$ \\
\hline CTA perfusion imaging & 0.752 & 0.618 & 0.686 & 0.692 & $4.90(3.33-7.22)$ & $2.23(1.79-2.77)$ & $<0.0001$ \\
\hline
\end{tabular}

LAMS, Los Angeles Motor Scale.

$\geq 4$ that were transported to either a primary stroke center or a thrombectomy-capable stroke center. Chart review confirmed that these 177 were patients whose time of onset was unknown or occurred $>24 \mathrm{~h}$ ago, in accordance with our stroke protocol. Of these, 161 were sent to a primary stroke center and 16 were sent to a thrombectomycapable stroke centers. Among these 177 patients, 31 were discharged home, 21 were discharged to a skilled nursing 
facility, 12 were discharged to rehabilitation, 12 expired, and 27 had unknown discharge disposition. By stroke type, 101 patients were diagnosed with ischemic stroke, 19 were diagnosed with hemorrhagic stroke, 5 were diagnosed with transient ischemic attack, and 47 had stroke mimics. In total, $66 \%$ of patients with high LAMS were transported to a comprehensive stroke center compared with $35 \%$ of those with low LAMS. Note that, especially for the patients with low LAMS, transport to a comprehensive stroke center may not have been due to suspected large vessel occlusion, but rather because the comprehensive stroke center is the closest stroke facility.

\section{Discussion}

To our knowledge, this is the largest study of a large vessel occlusion identification scale used in real time in the field by emergency medical services personnel for all suspected stroke calls. Thus, in addition to ischemic stroke, our cohort also included hemorrhagic strokes, transient ischemic attacks, and stroke mimics, which reflects the setting in which any prehospital large vessel occlusion prediction instrument would actually be used.

At the core of our stroke protocol is the patient, as is true of all of our Polk County Fire Rescue protocols. We developed the protocol based on the best evidence available and performed continuous quality assurance to ensure that we continue to give patients the best chance at the best recovery from stroke. In other words, our system preferentially transports those patients with more severe diseases to comprehensive stroke centers. To date, the estimated impact of our LAMS-based protocol is the transport of 10 more patients a month to a comprehensive stroke center rather than a primary stroke center.

Given the large sample size, the uniformity with which the assessments were conducted and completeness of field data capture followed by detailed outcome data, we were able to construct robust statistical models to test the performance of the LAMS as a tool to identify those who would benefit from being transported directly to a comprehensive stroke center.

Several studies have examined the performance of prehospital scales to determine transport decisions. Indeed, the LAMS has previously been shown to be correlated with large vessel occlusions $[23,24]$ several years ago. However, that early study was a convenience sample done using components derived from the National Institutes of Health Stroke Scale and by neurologists at bedside, which returns us back to the unanimous recommendation by several sys- tematic reviews [16-19] and a commentary [25] which emphasizes the need for prehospital stroke evaluation to be performed in the field by field personnel. One paper [26] studied the performance of various scales and proposed valuable scale features. These included binary scoring, inclusion of gaze deviation and arm weakness, exclusion of neglect, and prehospital validation in a suspected stroke cohort. Except for inclusion of gaze deviation, the LAMS tool in our cohort meets all of these criteria.

Mechanical intervention can be in the form of endovascular thrombectomy for large vessel occlusion strokes or coiling/clipping of cerebral aneurysms for hemorrhagic strokes. It is important to note that in the prehospital arena, distinguishing an ischemic from a hemorrhagic stroke is not the goal; rather, it is to identify which patients may need mechanical intervention and thus warrant transport to a comprehensive stroke center. Thus, the sensitivity and specificity for a scale should take into account both scenarios. In the current study, a LAMS of 4 was associated with the need for either endovascular thrombectomy or intervention for a hemorrhagic stroke. However, the PPV was $27 \%$ even with a LAMS of 5 , so one could argue that the majority of patients did not benefit from bypass to a comprehensive stroke center (the positive predictive value estimate would be higher if we knew the exact numbers of patients who received acute intervention for intracranial hemorrhage). This would support the recent preliminary findings reported by the RACECAT trial [27] which did not find benefit to bypass in Spain where the study was conducted. Our intent as emergency medicine field personnel is not to argue in favor or against bypass, but rather to share our real-world experience and allow policy makers to decide jurisdictional practices. For our part, we were looking to use an existing validated scale to make day-to-day decisions in the prehospital arena. We recognize this as a limitation of our study. It has been suggested that systems that use large vessel occlusion prediction instruments for prehospital triage will likely experience both under- and overtriage of large vessel occlusion stroke [28]. This depends on the prevalence of large vessel occlusion in the community and the local tolerance for under- and over-triage. In a community with low large vessel occlusion prevalence and a single comprehensive stroke center, for example, it may be reasonable to sacrifice sensitivity for specificity.

A key question is whether average benefit is greater with early thrombolysis at the closest primary stroke center before transportation to the comprehensive stroke center (Drip'n Ship) or with primary stroke center bypass and direct transport to the comprehensive stroke center. Model- 
ing studies suggest that a bypass strategy is warranted when a primary stroke center is close to a comprehensive stroke center unless the door-to-needle-time is $<30 \mathrm{~min}$ at the primary stroke center [29]. A more recent study [30] examined this time recommendation based on ideal versus real-world time metrics and whether an LVO was suspected based on prehospital large vessel occlusion scale result. It found that transport to a comprehensive stroke center was favored for $<32-99$ min for patients who screened positive for an LVO and $<28-39 \mathrm{~min}$ in the absence of such screening. A study of 234 patients in a large telestroke network found that longer transfer time was associated with lower rates of thrombectomy, and transfer at night rather than during the day was associated with significantly longer delay. The authors concluded that metrics and protocols for more efficient transfer, especially at night, could shorten transfer times [31]. For our local emergency medical services system, we felt that thorough protocolized triage on scene would be better than triage in the emergency department where desire of all hospitals to keep the patients in house once they arrive can result in unnecessary delay. Ultimately, decisions of whether to transport to a comprehensive stroke centers will depend on geography and local emergency medical services resources. Our study reports on the performance of the LAMS as a large vessel occlusion screening tool when used in a real-world setting.

\section{Conclusion}

In this prospective study of LAMS performed by emergency medical services personnel for all suspected stroke calls, the authors found that the LAMS is a useful prehospital predictor of intervention and outcomes after acute stroke. A LAMS of 4 or more is a predictor of a patient who could benefit from the services available at a comprehensive stroke center, whether that be thrombectomy for a large vessel occlusion or intervention for aneurysmal bleeding. Based on these data, the authors suggest consid- eration of transporting patients with a LAMS of 4 or more to a comprehensive stroke center.

\section{Acknowledgements}

T.G.S.: None. L.G. and P.B.: these authors also work as emergency physicians at a HCA healthcare facility, and although this was an entirely prehospital study with no funding or support from HCA, they are required to state the following: "This research was supported (in whole or in part) by HCA Healthcare and/or an HCA Healthcare affiliated entity. The views expressed in this publication represent those of the author(s) and do not necessarily represent the official views of HCA Healthcare or any of its affiliated entities."

\section{Statement of Ethics}

This study was approved by the University of Central Florida Institutional Review Board (Study \# SBE-17-13224). A waiver of informed consent was issued as the research was deemed no more than minimal risk, and that the study could not practicably be carried out without the waiver.

\section{Conflict of Interest Statement}

The authors have no conflicts of interest to disclose.

\section{Funding Sources} tion.

This study was not funded by any external entity or organiza-

\section{Author Contributions}

P.R.B. and L.G. conceived the study. T.S. and L.G. performed the statistical analyses and cross-checked the data. P.R.B. supervised the conduct of the research and data collection. L.G. and T.S. drafted the manuscript, and all the authors contributed substantially to its revision.

\section{References}

Field Performance of the Los Angeles Motor Scale
1 Benjamin EJ, Muntner P, Alonso A, Bittencourt MS, Callaway CW, Carson AP, et al. Heart disease and stroke statistics-2019 update: a report from the American Heart Association [published correction appears in Circulation. 2020 Jan 14;141(2):e33]. Circulation. 2019;139(10):e56-e528.

2 National Institute of Neurological Disorders and Stroke rt-PA Stroke Study Group. Tissue plasminogen activator for acute ischemic stroke. N Engl J Med. 1995;333(24):1581-7.
3 Powers WJ, Rabinstein AA, Ackerson T, Adeoye OM, Bambakidis NC, Becker K, et al. Guidelines for the early management of patients with acute ischemic stroke: 2019 update to the 2018 guidelines for the early management of acute ischemic stroke: a guideline for healthcare professionals from the American Heart Association/American Stroke Association [published correction appears in Stroke. 2019 Dec;50(12):e440-e441]. Stroke. 2019; 50(12):e344-e418. 
4 Hacke W, Kaste M, Bluhmki E, Brozman M, Dávalos A, Guidetti D, et al. Thrombolysis with alteplase 3 to 4.5 hours after acute ischemic stroke. N Engl J Med. 2008;359(13): 1317-29.

5 Nogueira RG, Jadhav AP, Haussen DC, Bonafe A, Budzik RF, Bhuva P, et al. Thrombectomy 6 to 24 hours after stroke with a mismatch between deficit and infarct. $\mathrm{N}$ Engl J Med. 2018;378(1):11-21.

6 Venema E, Duvekot MHC, Lingsma HF, Rozeman AD, Moudrous W, Vermeij FH, et al. Prehospital triage of patients with suspected stroke symptoms (PRESTO): protocol of a prospective observational study. BMJ Open. 2019;9(7):e028810.

7 Saver JL, Goyal M, van der Lugt A, Menon BK, Majoie CB, Dippel DW, et al. Time to treatment with endovascular thrombectomy and outcomes from ischemic stroke: a meta-analysis. JAMA. 2016;316(12):1279-88.

8 Chenaitia H, Lefevre O, Ho V, Squarcioni C, Pradel V, Fournier M, et al. Emergency medical service in the stroke chain of survival. Eur J Emerg Med. 2013;20(1):39-44.

9 Alberts MJ, Latchaw RE, Selman WR, Shephard T, Hadley MN, Brass LM, et al. Recommendations for comprehensive stroke centers: a consensus statement from the Brain Attack Coalition. Stroke. 2005 Jul;36(7): 1597-616.

10 Leifer D, Bravata DM, Connors JJ 3rd, Hinchey JA, Jauch EC, Johnston SC, et al. Metrics for measuring quality of care in comprehensive stroke centers: detailed follow-up to brain attack coalition comprehensive stroke center recommendations: a statement for healthcare professionals from the American Heart Association. Stroke. 2011 Mar; 42(3):849-77.

11 McKinney JS, Cheng JQ, Rybinnik I, Kostis JB; Myocardial Infarction Data Acquisition System (MIDAS 22) Study Group. Comprehensive stroke centers may be associated with improved survival in hemorrhagic stroke. J Am Heart Assoc. 2015;4(5):e001448.
12 Singer AJ, Thode HC Jr, Viccellio P, Pines JM. The association between length of emergency department boarding and mortality. Acad Emerg Med. 2011 Dec;18(12):1324-9.

13 Kulstad EB, Sikka R, Sweis RT, Kelley KM, Rzechula KH. ED overcrowding is associated with an increased frequency of medication errors. Am J Emerg Med. 2010;28(3):304-9.

14 Pines JM, Pollack CV Jr, Diercks DB, Chang AM, Shofer FS, Hollander JE. The association between emergency department crowding and adverse cardiovascular outcomes in patients with chest pain. Acad Emerg Med. 2009;16(7):617-25.

15 Jo S, Kim K, Lee JH, Rhee JE, Kim YJ, Suh GJ, et al. Emergency department crowding is associated with 28 -day mortality in community-acquired pneumonia patients. J Infect. 2012;64(3):268-75

16 Zhelev Z, Walker G, Henschke N, Fridhandler J, Yip S. Prehospital stroke scales as screening tools for early identification of stroke and transient ischemic attack. Cochrane Database Syst Rev. 2019 Apr 9;4: CD011427.

17 Smith EE, Kent DM, Bulsara KR, Leung LY, Lichtman JH, Reeves MJ, et al. Accuracy of prediction instruments for diagnosing large vessel occlusion in individuals with suspected stroke: a systematic review for the 2018 guidelines for the early management of patients with acute ischemic stroke. Stroke. 2018;49: e111-22.

18 Krebs W, Sharkey-Toppen TP, Cheek F, Cortez E, Larrimore A, Keseg D, et al. Prehospital stroke assessment for large vessel occlusions: a systematic review. Prehosp Emerg Care. 2018 Mar-Apr;22(2):180-8.

19 Vidale S, Agostoni E. Prehospital stroke scales and large vessel occlusion: a systematic review. Acta Neurol Scand. 2018 Jul;138(1):2431.

20 Llanes JN, Kidwell CS, Starkman S, Leary MC, Eckstein M, Saver JL. The Los Angeles Motor Scale (LAMS): a new measure to characterize stroke severity in the field. Prehosp Emerg Care. 2004;8:46-50.
21 Stead TG, Banerjee PR, Ganti L. Large vessel occlusion identification through prehospital administration of stroke scales: a county-wide emergency medical services prospective research protocol. Cureus. 2019 Oct 17;11(10): e5931.

22 JMP Pro ${ }^{\circ}$. Version 14.1. Cary, NC: SAS Institute Inc.;1989-2019.

23 Nazliel B, Starkman S, Liebeskind DS, Ovbiagele B, Kim D, Sanossian N, et al. A brief prehospital stroke severity scale identifies ischemic stroke patients harboring persisting large arterial occlusions. Stroke. 2008 Aug; 39(8):2264-7.

24 Brandler ES, Thode H, Fiorella D. The Los Angeles Motor Scale as a predictor of angiographically determined large vessel occlusion. Intern Emerg Med. 2020 Jun;15(4):695-700.

25 Pérez de la Ossa N, Ribó M, Jiménez X, Abilleira S. Prehospital scales to identify patients with large vessel occlusion: it is time for action. Stroke. 2016 Nov;47(11):2877-8

26 Keenan KJ, Kircher C, McMullan JT. Prehospital prediction of large vessel occlusion in suspected stroke patients. Curr Atheroscler Rep. 2018 May 21;20(7):34.

27 Available from: https://clinicaltrials.gov/ct2/ show/NCT02795962 Accessed 2021 Jan 11.

28 Ganti L, Oostema JA. How accurate are the stroke severity scales for identifying large vessel occlusions? Ann Emerg Med. 2020 Apr; 75(4):494-6.

29 Milne MS, Holodinsky JK, Hill MD, Nygren A, Qiu C, Goyal M, et al. Drip 'n ship versus mothership for endovascular treatment: modeling the best transportation options for optimal outcomes. Stroke. 2017 Mar;48(3): 791-4.

$30 \mathrm{Xu}$ Y, Parikh NS, Jiao B, Willey JZ, Boehme AK, Elkind MSV. Decision analysis model for prehospital triage of patients with acute stroke. Stroke. 2019;50(4):970-7.

31 Regenhardt RW, Mecca AP, Flavin SA, Boulouis G, Lauer A, Zachrison KS, et al. Delays in the air or ground transfer of patients for endovascular thrombectomy. Stroke. 2018 Jun;49(6):1419-25. 